\title{
Resolving actions of compact Lie groups
}

\author{
M.J. Field
}

A general process for the desingularization of smooth actions of compact lie groups is described. If $G$ is a compact lie group, it is shown that there is naturally associated to any compact $G$ manifold $M$ a compact $G \times(z / 2)^{p}$ manifold on which $G$ acts principally. Here $Z / 2$ denotes the cyclic group of order two and $p+1$ is the number of orbit types of the $G$ action on $M$.

\section{Introduction}

Let $G$ be a compact Lie group. In this note we show that there is naturally associated to any compact $G$ manifold $M$ a compact $G \times(2 / 2)^{p}$ manifold $\hat{M}$ on which $G$ acts principally. Here $Z / 2$ denotes the cyclic group of order two and $p+1$ is the number of orbit types of the action of $G$ on $M$ (see $\S 2$ ). We call $\hat{M}$ a resolution of (the $G$ action on) $M$. Our method of construction of $\hat{M}$ is a modification of the familiar "blowing up" transformation of algebraic geometry and is closely related to the polar coordinate transformation as used, for example, by Ruelle and Takens in [4].

Our process of resolution is basic to a study of ours on the linearizations, modulo $G$, of equivariant diffeomorphisms close to the identity map and the construction of a maximal family of slices for a $G$ action. However, we feel that our results on resolutions may be of wider interest, with possible applications to the classification theory of smooth actions, and so we are presenting them separately.

Received 5 January 1978. 


\section{Blowing up $G$ manifolds}

We start by recalling some facts about smooth (that is $C^{\infty}$ ) actions of a compact Lie group on a compact differential manifold. We refer to Bredon's text [2], especially Chapter 6, for full details and proofs.

Given a compact connected differential manifold $M$ and the action of a compact lie group $G$ on $M$, we let $G(x)$ denote the $G$ orbit through $x$ and $G_{x}$ the isotropy subgroup of $G$ at $x, x \in M . G(x)$ is equivariantly diffeomorphic to the homogeneous space $G / G_{x}$. The isotropy subgroup at $g x, g \in G$, is conjugate to that at $x$ and indeed is obviously equal to $g G_{x^{g}} g^{-1}$. We say that $x, y \in M$ are of the some orbit type if $G_{x}$ and $G_{y}$ are conjugate subgroups of $G$ or, equivalently, if $G(x)$ and $G(y)$ are equivariantly diffeomorphic. The equality of orbit type partitions $M$ into points of the same orbit type. If $M$ is compact, this partition is finite. We write

$$
M=\underset{i \in I}{U} M_{i}
$$

where $M_{i}$ are the equivalence classes of points of the same orbit type. We define orb $: M \rightarrow I$ by $\operatorname{orb}(x)=i, x \in M_{i}$.

There is defined a natural partial order on $I$ by $i<j$ if there exist $x \in M_{i}, y \in M_{j}$, such that $G_{x} \supset G_{y}$ (strict inclusion). We say that $x$ is of minimal orbit type if there does not exist $y \in M$ such that $\operatorname{orb}(y)<\operatorname{orb}(x)$. The finiteness of $I$ implies there exists at least one minimal orbit type. We may similarly define a maximal orbit type. In this case it may be shown that there exists precisely one maximal orbit type, say $N$, and that $M_{N}$ is an open dense subset of $M$ (connected if $G$ is connected).

For convenience we shall label orbit types by integers and write

$$
M=\underset{1 \leq i \leq N}{U} M_{i} \text {, }
$$

where $\operatorname{orb}(x)<\operatorname{orb}(y)$ implies that if $x \in M_{i}$ and $y \in M_{j}$ then $i<j$ (the converse need not be true: if $i<j$ (as integers) then $G_{x}$ and $G_{y}$ 
need not be related. For example, $M_{1}$ and $M_{2}$ may both be minimal orbit types). We shall say that the action is principal if there exists only one orbit type and that it is free if $G_{x}=\{e\}$ for all $x \in M$.

Let $\xi$ be a riemannian metric on $M$. Averaging over $G$ using Haar measure we may assume that $\xi$ is $G$ invariant. We call $M$, together with a $G$ action and equivariant riemannian metric, a riemannian $G$ manifold. In the sequel, we assume $M$ is a riemannian $G$ manifold. Apart from the notation introduced above, we let $\operatorname{diff}_{G}^{k}(M)$ denote the space of $c^{k}$ equivariant diffeomorphisms of $M, 1 \leq k \leq \infty$. In case $k<\infty$, we give $\operatorname{diff}_{G}^{k}(M)$ the $C^{k}$ topology ([1], [3]).

DEFINITION. A resolution of $M$ consists of a $G \times(z / 2)^{N-1}$ manifold $\hat{M}$ and a $C^{\infty} \operatorname{map} \pi: \hat{M} \rightarrow M$ and homomorphism $\phi: \operatorname{diff}_{G}^{\infty}(M) \rightarrow \operatorname{diff}^{\infty}(\hat{M})$ such that:

(1) if we give $M$ the trivial $(2 / 2)^{N-1}$ action, $\pi$ is $G \times(2 / 2)^{N-1}$ equivariant;

(2) the generators $f_{1}, \ldots, f_{N-1}$ of the $(2 / 2)^{N-1}$ action on $\hat{M}$ may be indexed so that

$$
\begin{aligned}
\pi^{-1}\left(M_{j}\right) & =\operatorname{fix}\left(f_{j}\right) \backslash \underset{i<j}{\cup} \operatorname{fix}\left(f_{i}\right), 1 \leq j \leq N-1, \\
& =\text { free part of the }(z / 2)^{N-1} \text { action, } j=N ;
\end{aligned}
$$

(3) $G$ acts principally on $\hat{M}$;

(4) for all $f \in \operatorname{diff}_{G}^{\infty}(M), \phi(f)$ is a $C^{\infty} G \times(2 / 2)^{N-1}$ invariant map covering $f$.

REMARK. It follows from (2) of the definition that $\pi / \pi^{-1}\left(M_{N}\right)$ is a $2^{N-1}$ fold covering map of $M_{N}$.

THEOREM A. Every compact $G$ manifold has a resolution. Moreover, for the resolution we constmuct we may require that the extension map 
$\phi: \operatorname{diff}_{G}^{\infty}(M) \rightarrow \operatorname{diff}^{\infty}(\hat{M})$ extends to a continuous map

$$
\phi: \operatorname{diff}_{G}^{k+N-1}(M) \rightarrow \operatorname{diff}^{k}(\hat{M}), \quad r \geq 0 .
$$

Before starting the proof of Theorem A, we prove a simple and presumably well known result which is special to actions by finite groups of odd order.

THEOREM B. Let $G$ be a finite group of odd order acting on $M$. Then there exists a principal $G$ manifold $\tilde{M}$ and $a C^{\infty}$ equivariont map $\pi: \tilde{M} \rightarrow M$ such that $\pi^{-1}\left(M_{N}\right)$ is open and dense in $\tilde{M}$ and $\pi$ mops $\pi^{-1}\left(M_{N}\right)$ diffeomorphically onto $M_{N}$.

Proof. We shall successively blow up the submanifolds $M_{1}, \ldots, M_{N-1}$. The techniques we use are well known and standard in equivariant differential topology and so we only outline the main details. Let $E_{1} \rightarrow M_{1}$ denote the normal bundle of $M_{1}$ and choose $r>0$ so that the disc bundle $E_{I}(r)=\left\{v \in E_{I}:\|v\|<r\right\}$ is embedded as a tubular neighbourhood $Q(r)$ of $M_{1}$ by the exponential map. Choosing $r$ smaller if necessary, we may also require that $\partial Q(r)$ is a codimension one submanifold of $M$ equivariantly diffeomorphic to the unit sphere bundle $S\left(E_{1}\right)$ of $E_{1}$. Define $\gamma: S\left(E_{1}\right) \times R \rightarrow R$ by $\gamma(\theta, t)=\exp (t \theta) \cdot Z / 2$ acts freely on $S\left(E_{1}\right) \times R$ as multiplication by -1 (on both factors) and this action commutes with $Y$. If $X$ is any $Z / 2$ invariant subset of $R$, we let $P\left(E_{1}, X\right)$ denote the orbit space of the induced $Z / 2$ action on $S\left(E_{1}\right) \times X \cdot \gamma$ restricts to a $C^{\infty}$ diffeomorphism of $P\left(E_{1},\{-r,+r\}\right)$ with $\partial Q(r)$ and, in the usual way, we may form the $G$ manifold

$$
\tilde{M}_{1}=(M \backslash Q(r)) \underset{\gamma}{\cup} P\left(E_{I},[-r,+r]\right) .
$$

$\pi_{1}: \tilde{M}_{1} \rightarrow M$ is defined to be the identity on $M \backslash Q(p)$ and $\gamma$ on $P\left(E_{1},[-r,+r]\right)$. Clearly $\pi_{1}$ is a diffeomorphism of $\pi_{1}^{-1}\left(M_{1}\right)$. Since $G$ is of odd order, it does not contain any $z / 2$ subgroups. Hence the orbit types of $G$ on $P\left(E_{1},[-r,+r]\right)$ are the same as those of $G$ on 
$S\left(E_{1}\right] \times[-r,+r]$, which in turn are a subset of the orbit types $2, \ldots, N$. Hence no orbit of type 1 appears in $\tilde{M}_{1}$. Iterating this process we may remove orbits of types 2 up to $N-1$. //

Proof of Theorem A. As in the proof of Theorem B, we form the unit sphere bundle $S\left(E_{1}\right)$ of the normal bundle $E_{1}$ of $M_{1}$ and choose $r>0$ so that exp embeds the disc bundle of radius $r^{2}$ as a tubular neighbourhood $Q(r)$ of $M_{1}$ with smooth boundary $\partial Q(r)$. Let $\gamma: S\left(E_{1}\right) \times R \rightarrow M$ be the map $(\theta, t) \mapsto \exp \left(t^{2} \theta\right)$. $\gamma$ is $G \times(z / 2)$ invariant if we take the $z / 2$ action on $S\left(E_{1}\right) \times R$ defined by $(\theta, t) \rightarrow(\theta,-t)$ and the trivial $Z / 2$ action on $M$. We define $\hat{H}_{1}$ to be

$$
(M Q(r)) \underset{\gamma_{-}}{U}\left(S\left(E_{1}\right) \times[-r,+r]\right) \underset{\gamma_{+}}{U}(M Q(r)),
$$

where $\gamma_{ \pm}=\gamma \mid S\left(E_{1}\right) \times\{ \pm r\}$ identifies $\partial Q(r)=\partial(M \backslash Q(r))$ to $S\left(E_{1}\right) \times\{ \pm r\}$. The $Z / 2$ action on $S\left(E_{1}\right) \times[-r,+r]$ extends in the obvious way to $\hat{M}_{1}$ and, since $\gamma$ is $G \times(z / 2)$ invariant, we see that $\hat{M}_{1}$ is a $G \times(z / 2)$ manifold. $\pi_{1}: \hat{M}_{1} \rightarrow M$ is defined to be the identity on either of the components $M Q(r)$ and $\gamma$ on $S\left(E_{1}\right) \times[-r,+r]$. Set

$$
M_{1}^{ \pm}=S\left(E_{1}\right) \times(0, \pm r] \underset{\gamma_{ \pm}}{\bigcup}(M Q Q(r)) .
$$

$\pi_{1}$ restricts to an equivariant diffeomorphism $\pi_{1}^{ \pm}$of $M_{1}^{ \pm}$onto $M M_{1}$. The only orbit types that can occur for the $G$ action on $\hat{M}_{1}$ are $2, \ldots, N$. If we let $\alpha_{1}^{1}$ be the involution generating the $2 / 2$ action on $\hat{M}_{1}, \alpha_{1}$ is $C^{\infty}$, equivariant and has fixed point set $\pi_{1}^{-1}\left(M_{1}\right)$. Let $f \in \operatorname{diff}_{G}^{k}(M)$. Then $f\left(M_{j}\right)=M_{j}, j=1, \ldots, N$. We define $\hat{f}_{1}: \hat{M}_{1}^{ \pm} \rightarrow M_{1}^{ \pm}$by

$$
\hat{f}_{1}=\left(\pi_{1}^{ \pm}\right)^{-1} \pi_{1}^{ \pm}
$$

Clearly $f_{1}$ is $C^{k}$ on $M_{1}^{ \pm}$. Choose $s>0$ so that $f(Q(s)) \subset Q(r)$. 
Suppose $s<r$. Then for $0<|t|<s$, we see that, relative to the coordinates on $\hat{M}_{1}$ given by $S\left(E_{1}\right) \times[-r,+r]$,

$$
\hat{f}_{1}(t, \theta)=\left\{\frac{\exp ^{-1}\left(f \exp \left(t^{2} \theta\right)\right)}{\left\|\exp ^{-1}\left(f \exp \left(t^{2} \theta\right)\right)\right\|}, \operatorname{sign}(t)\left\|\exp ^{-1}\left(f \exp \left(t^{2} \theta\right)\right)\right\|^{\frac{1}{2}}\right) \text {. }
$$

$\hat{f}_{1}$ is certainly $G \times(z / 2)$ invariant. We claim that $\hat{f}_{1}$ extends as a $c^{k-1} G \times(2 / 2)$ invariant map across $\pi_{1}^{-1}\left(M_{1}\right)$. For this it is clearly enough to show that there exists a $c^{k-1} \operatorname{map} g: S\left(E_{1}\right) \times(-8,+s) \rightarrow E_{1}$ such that $g \neq 0$ and

$$
\exp ^{-1}\left(f \exp \left(t^{2} \theta\right)\right)=t^{2} g(\theta, t), \quad t \neq 0, \theta \in s\left(E_{1}\right) \text {. }
$$

Fix a $C^{\infty}$ embedding of $M$ into some $R^{n}$. Such an embedding induces an embedding of $T M$ in $R^{2 n}$ and, by restriction, of $E_{1}$ into $R^{2 n}$. For $\theta \in S\left(E_{l}\right)$, consider the map $\rho_{\theta}:(-s,+8) \rightarrow R^{2 n}$ defined by

$$
\rho_{\theta}(t)=\exp ^{-1}(f \exp (t \theta))
$$

Then

$$
\begin{aligned}
\rho_{\theta}\left(t^{2}\right) & =\int_{0}^{1} \frac{\partial}{\partial u}\left(\rho_{\theta}\left(u t^{2}\right)\right) d u \\
& =t^{2} \int_{0}^{1} \rho_{\theta}\left(u t^{2}\right) d u .
\end{aligned}
$$

Therefore, if we define $g(\theta, t)=\int_{0}^{1} \rho_{\theta}\left(u t^{2}\right) d u, g$ will satisfy our requirements. Moreover, it is easily verified that if $\left(f_{n}\right)$ is a convergent sequence in the $c^{k}$ topology, the corresponding sequence $\left(g_{n}\right)$ will be convergent in the $c^{k-1}$ topology. In other words the map $\operatorname{diff}_{G}^{k}(M)+\operatorname{diff}^{k-1}\left(\hat{M}_{1}\right) ; f \rightarrow \hat{f}_{1}$ is continuous. The map is obviously a homomorphism. 
Suppose inductively that we have performed $j$ successive polar blow ups to obtain a $G \times(Z / 2)^{j}$ manifold $\hat{M}_{j}$ with $G$ orbit types $j+1, \ldots, N, j+1<N$. Denote the generators of the $(z / 2)^{j}$ action by $\alpha_{j}^{1}, \ldots, \alpha_{j}^{j}$. As above we choose an equivalent riemannian metric on $\hat{M}_{j}$ and polar blow up the set of points of orbit type $j+1$ to obtain a new $G$ manifold $\hat{M}_{j+1}$. We set $\alpha_{j+1}^{i}=\hat{\alpha}_{j}^{i}, 1 \leq i \leq j$, and let $\alpha_{j+1}^{j+1}$ denote the generator of the $z / 2$ action originating from the polar blow up of $\hat{M}_{j}$. Now since $\alpha_{j}^{i}$ is $G$ invariant $\alpha_{j+1}^{i}$ commutes with $\alpha_{j+1}^{j+1}$, $1 \leq i \leq j$. Also $\alpha_{j+1}^{i}$ and $\alpha_{j+1}^{k}$ commute for $1 \leq i, k \leq j$ since the lifting map is a homomorphism. Hence we have a $G \times(2 / 2)^{j+1}$ action of $\hat{M}_{j+1}$ with $\alpha_{j+1}^{1}, \ldots, \alpha_{j+1}^{j+1}$ generators of the $(2 / 2)^{j+1}$ action.

Similarly, if we have shown inductively that a $C^{k} G$ diffeomorphism $f$ of $M$ lifts to a $C^{k-j} G \times(Z / 2)^{j}$ diffeomorphism $\hat{f}_{j}$ of $\hat{M}_{j}$, then $\hat{f}_{j}$ lifts to a $c^{k-j-1}$ diffeomorphism $\hat{f}_{j+1}$. of $\hat{M}_{j+1}$. Hence the inductive step is completed and we may take $\hat{M}=\hat{M}_{N-1}, \phi(f)=\hat{f}_{N-1}$. The generators of the $(Z / 2)^{N-1}$ action on $\hat{M}$ are $\alpha_{N-1}^{j}, 1 \leq j \leq N-1 . \quad / /$

REMARKS. In the sequel we shall refer to the resolution of $M$ constructed in Theorem A as the polar resolution of $M$. We call the manifold $\hat{M}_{j}$ obtained in the proof of Theorem A after $j$ successive polar blow ups the $j$-fold poler blow up of $M$. We denote the $i$ th orbit type of $\hat{M}_{j}$ by $\left(\hat{M}_{j}\right)_{i}$. Thus we will have $\left(\hat{M}_{j}\right)_{i}=\emptyset, 1 \leq i \leq j$, and $\hat{M}_{N-1}=\left(\hat{M}_{N-1}\right)_{N-1}=\hat{M}$.

EXAMPLE. Take the circle action on $S^{3}$ induced from scalar multiplication on $c^{2}$ by $\left(e^{p i \phi}, e^{q i \phi}\right), p, q \in z^{+}$. If $p=q$, the action on $s^{3}$ is principal. If $p \mid q$ or $q \mid p, p \neq q$, then there are two orbit types with corresponding isotropy subgroups $2 / p, 2 / q$. Suppose that $q>p$. Then the minimal orbit type consists of a single 
$S^{1}$ orbit with isotropy subgroup $2 / q$. Resolving, we find

$$
\hat{s}^{3}=D^{2} \times S^{1} \underset{\text { id }}{\cup} D^{2} \times s^{1}=s^{2} \times S^{1} .
$$

The circle action on $D^{2} \times S^{\perp}$ is $(z, y) \mapsto\left(z e^{i q \phi}, y e^{i p \phi}\right), z \in D^{2}$, $y \in S^{1} \subset C$, and the involution is reflection in $\partial D^{2} \subset S^{2}$ with fixed set $\partial D^{2} \subset S^{1}=T^{2}$.

Finally suppose $p / q, q$. There are now three orbit types with isotropy subgroups $z / p, Z / q, Z / m$, where $m$ is the highest common factor of $p$ and $q$. In this case $\hat{S}^{3}=T^{3}$ and the circle action on

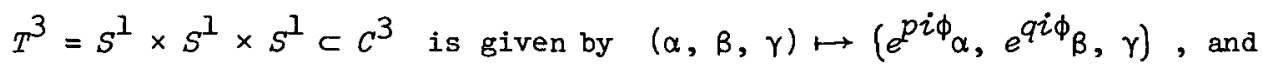
the involutions are

$$
\begin{aligned}
& f_{1}(\alpha, \beta, \gamma)=(\alpha, \beta, \bar{\gamma}), \\
& f_{2}(\alpha, \beta, \gamma)=(\alpha, \beta,-\bar{\gamma}) .
\end{aligned}
$$

LEMMA C. Up to $G \times(z / 2)^{j}$ diffeomorphism, the manifolds $\hat{M}_{j}$ constructed in the proof of Theorem A are independent of choices of riemannian metrics on $M, \ldots, \hat{M}_{j-1}$.

Proof. It is enough to prove this for $j=1$, since the general case follows by iteration. Let $M$ have equivariant riemannian metrics $\xi$ and $\xi^{\prime}$. Following the notation and assumptions of the proof of Theorem $A$, we define

$$
\gamma_{1}: S\left(E_{1}\right) \times[-r,+r] \rightarrow S\left(E_{1}\right)^{\prime} \times R
$$

by

$$
\gamma_{1}(\theta, t)=\left(H\left(t^{2} \theta\right) /\left\|H\left(t^{2} \theta\right)\right\|^{\prime}, \operatorname{sign}(t)\left(\left\|H\left(t^{2} \theta\right)\right\|^{\prime}\right)^{\frac{3}{2}}\right)
$$

where $H\left(t^{2} \theta\right)=\left(\exp ^{\prime}\right)^{-1}\left(\exp \left(t^{2} \theta\right)\right)$ and $\exp ^{\prime},\|\|^{\prime}$, and $s\left(E_{1}\right)^{\prime}$ are the exponential norm and unit sphere bundle corresponding to $\xi^{\prime}$. As in Theorem A, $\gamma_{1}$ is $c^{\infty}$. Taking $r$ smaller if necessary, we may assume that the image of $\gamma_{1}$ lies in $S\left(E_{1}\right)^{\prime} \times\left[-r^{\prime},+r^{\prime}\right]$. We extend $\gamma_{1}$ to a 
$G \times(2 / 2)$ diffeomorphism of $\hat{M}_{1}$ by setting $\gamma_{1}=\left(\pi_{1}\right)^{ \pm}\left(\pi_{1}\right)^{ \pm}$outside $S\left(E_{1}\right) \times[-r,+r]$. //

COROLLARY. The polar resolution of $M$ is independent of the choice of metries up to $G \times(Z / 2)^{N-1}$ diffeomorphism.

\section{Blowing down $G$ manifolds}

It is not hard to prove a converse to Theorem $A$ and in this final section we shall indicate how this may be done.

DEFINITION. A $G$ sphere bundle is a quadruple $(X, E, \Sigma, \rho)$ consisting of a riemannian $G$ vector bundle $\pi: E \rightarrow \Sigma$, where $\Sigma$ is a principal $G$ manifold, and an equivariant diffeomorphism $\rho: S(E) \rightarrow X$, where $S(E)$ is the unit sphere bundle of $E$. We usually refer to the " $G$ sphere bundle $X$ ".

Let $\pi: E \rightarrow \Sigma$ be a riemannian $G$ vector bundle and $N(S(E))$ denote the normal bundle of $S(E)$ in $E$. Clearly $N(S(E))$ is a trivial line bundle over $S(E)$. $N(S(E))$ has a natural $2 / 2$ action induced by scalar multiplication by -1 in the fibres and the action has fixed set $S(E)$ the zero section of $N(S(E))$. Since the $Z / 2$ action commutes with the $G$ action on $N(S(E))$, we see that $N(S(E))$ has the structure of a $G \times(z / 2)$ bundle over $S(E)$. If we take the product of the standard $2 / 2$ action on $R$ with the $G$ action on $S(E)$, then $S(E) \times R$ and $N(S(E))$ are isomorphic as $G \times(2 / 2)$ bundles.

PROPOSITION. Let $N$ be a compact connected $G \times(z / 2)$ manifold and $f$ be the generator of the $Z / 2$ action on $N$. Suppose that

(1) $\operatorname{fix}(f)$ is a $G$ sphere bundle; that is $f i x(f)$ is associated to a quadmule $(\operatorname{fix}(f), E, \Sigma, \rho)$;

(2) $\rho^{*}(N(f i x(f)))$ and $N(S(E))$ are isomorphic as $G \times(Z / 2)$ bundies,

(3) $\quad M$ fix $(f)$ has two connected components $N_{1}, N_{2}$, and $f\left(N_{1}\right)=N_{2}$.

Then there exists a unique, up to $G$ diffeomorphism, $G$ manifold $M$ such that $N$ is $G \times(z / 2)$ diffeomorphic to the polar blow up of $M$ 
along a minimal orbit type.

Proof. Essentially a reversal of the argument of Theorem A. Fix $a>0$ and give $N$ an equivariant riemannian metric. Since $S(E) \times R$ and $\rho^{*} N(f i x(f))$ are isomorphic as $G \times(z / 2)$ bundles, there exists a $G \times(Z / 2)$ diffeomorphism $\gamma$ of $S(E) \times(-a,+a)$ onto a tubular neighbourhood $Q$ of $\operatorname{fix}(f)$. Here we suppose that $Q$ has smooth boundary which is the image of a sphere bundle of $N(f i x(f))$ by the exponential map. Regarding $\Sigma$ as the zero section of $E$, the normal bundle of $\Sigma$ is isomorphic to $E$ as a $G$ bundle and consequently, polar blown up along $\Sigma$ is $G \times(z / 2)$ diffeomorphic to $Q$. We now construct the required manifold $M$ by identifying the boundaries of $N_{1} \backslash Q$ and the disc bunde of $E$ of radius a using the map $\gamma$. //

Suppose $(X, E, \Sigma, \rho)$ is a $G$ sphere bundle. We may resolve the $G$ space $X$ to $\hat{X}$ as in Theorem A. If $X$ has $r$ orbit types, this will require $r-1$ steps and $\hat{X}$ will be a $G \times(z / 2)^{r-1} \operatorname{manifold}$ on which $G$ acts principally. We call $\hat{X}$ the "resolved $G$ sphere bundle $(X, E, \Sigma, \rho) "$. We let $\widehat{N(S(E))}$ and $\widehat{S(E)}$ denote the polar resolutions of $N(S(E))$ and $S(E)$ respectively. Since $N(S(E)) \cong S(E) \times R$, $N(S(E)) \cong \widehat{S(E)} \times R$ and $\widehat{S(E)}$ is of codimension one in $N(S(E))$. In case we have a $(2 / 2)^{q}$ action on $(X, E, \Sigma, \rho)$ which commutes with $G$, we shall refer to $X$ as a resolved $G \times(z / 2)^{q}$ sphere bundle, it being understood that we do not resolve the $(z / 2)^{q}$ action.

Given a $G \times(z / 2)^{p}$ action on $N$, suppose that $\left\{f_{1}, \ldots, f_{p}\right\}$ is the set of generators for the $(z / 2)^{p}$ action. Observe that $\operatorname{fix}\left(f_{j}\right)$ is left invariant by $G \times(z / 2)^{p}$. It follows that $N\left(\operatorname{fix}\left(f_{j}\right)\right)$ has the structure of a $G \times(z / 2)^{p}$ bundle over $\operatorname{fix}\left(f_{j}\right), 1 \leq j \leq p$.

THEOREM D. Let $N$ be a compact connected $G \times(z / 2)^{p}$ manifold on which $G$ acts principally. Suppose that we can find an ordering $\left\{f_{1}, \ldots, f_{p}\right\}$ of the set of generators of the $(z / 2)^{p}$ action such that

(1) each submanifold fix $\left(f_{j}\right)$ is a resolved $G \times(z / 2)^{j}$ 
sphere bundze $\left(x_{j}, E_{j}, \Sigma_{j}, \rho_{j}\right)$, and the generators of the $(z / 2)^{j-1}$ action are $\left(f_{1}, \ldots, f_{j-1}\right)$,

(2) $N\left(S\left(E_{j}\right)\right)$ and $N\left(\right.$ fix $\left.\left(f_{j}\right)\right)$ are isomorphic as $G \times(Z / 2)^{p}$ bundies,

(3) $N \backslash \bigcup_{i=j}^{p} \operatorname{fix}\left(f_{i}\right)$ has $2^{p-j+1}$ connected components $N_{u}^{j}$, $1 \leq u \leq 2^{p-j+1}$, and $\left\{f_{j}, \ldots, f_{p}\right\}$ acts transitively on the set of components and, given $s, 1 \leq s \leq p$, each $N_{u}^{j}$, $j<s$, is contained wholly within some $N_{k}^{s}$.

Then there exists a unique, up to $G$ diffeomorphism, $G$ manifold $M$ such that $N$ is $G \times(2 / 2)^{p}$ diffeomorphic to the polar resolution of $M$.

Proof. The proof follows straightforwardly by repeated application of the proposition and we omit details.

REMARKS. 1. If $\left(X_{j}, E_{j}, \Sigma_{j}, \rho_{j}\right)$ has less than $p-j+1$ orbit types we nevertheless resolve $p-j$ times, doubling up when the orbit type is empty.

2. Since $S(E)$ is of codimension one in $N(S(E))$, condition (2) of Theorem $D$ implies that $\operatorname{fix}\left(f_{j}\right)$ is of codimension one, $1 \leq j \leq p$.

3. We require $N$ to be connected to avoid exceptional cases where $M$ is a $G$ manifold with a minimal orbit type of codimension one and trivial normal bundle. In such cases the polar resolution of $M$ ceases to be connected. We leave the formulation of the appropriate version of Theorem $D$ to the reader.

4. Theorem D implies that if $M$ and $M^{\prime}$ have $G \times(z / 2)^{p}$ diffeomorphic polar resolutions then $M$ is $G$ diffeomorphic to $M^{\prime}$. 


\section{References}

[1] Ralph Abraham, Joel Robbin, Transversal mappings and flows (Benjamin, New York, Amsterdam, 1967).

[2] Glen E. Bredon, Introduction to compact tronsformation groups (Pure and Applied Mathematics, 46. Academic Press, New York and London, 1972).

[3] M. Golubitsky, V. Guillemin, Stable mappings and their singularities (Graduate Texts in Mathematics, 14. Springer-Verlag, New York, Heidelberg, Berlin, 1973).

[4] David Ruelle and Florio Takens, "On the nature of turbulence", Comm. Hath. Phys. 20 (1971), 167-192.

Department of Pure Mathematics, University of Sydney,

Sydney,

New South Wales. 